\title{
A NEW CLOSED FORM SOLUTION FOR DYNAMIC STABILITY ANALYSIS OF ROLLING AIRFRAMES HAVING ONE PAIR ON-OFF ACTUATOR
}

\author{
Jalal KARIMI* \\ Aerospace Engineering Department, Malek-Ashtar University of Technology, Tehran, Iran
}

Received 21 September 2020; accepted 30 September 2020

\begin{abstract}
In this paper, the dynamic stability analysis of a rolling airframe actuated by one pair ON-OFF actuator using linear theory is presented via developing a new closed form solution. The effect of discontinuous forcing term on rolling airframe stability is studied. In contrast to tricyclic motion with constant forcing term (constant non-homogeneous term) in which only the amplitude of nutation and precession is affected, it is found that ON-OFF control affects both amplitude and phase of nutation and precession motions. In the case of discontinuous control surface, there are two sources for resonance instability. Finally, through simulation results of closed form solutions, a comparison between airframe' response to ideal and real ON-OFF command is achieved. The effect of ON-OFF control on angular motion is also evaluated.
\end{abstract}

Keywords: rolling airframe, one pair ON-OFF actuator, dynamic stability, resonance instability, linear analysis, closed form solution.

\section{Introduction}

During the last few decades, the use of rolling airframes is increased. The main benefit of rolling motion is overcoming the effect of airframe asymmetries due to thrust or fins misalignment. One pair control surface with ONOFF actuator is one of the complicated configurations for analytical analysis. The advantage of using one pair control surface is to reduce airframe size, mass and price. In general, DC motor and ON-OFF actuators are used to move control surfaces. It is known that ON-OFF actuator is a non-linear element and an additional signal, which called linearity or dither signal, is used for linearization its output. In this paper the dynamic stability of a rolling airframe with one pair of control surface actuated by $\mathrm{ON}-\mathrm{OFF}$ actuator is addressed.

The equations of motion of rolling airframe in bodyfixed frame and in non-rolling frame are developed in literature (Nicolaides, 1953; Murphy, 1963, 1981, 1971; Cohen et al., 1974). Relying on these models, the stability analysis of free flight motion and the effect of small asymmetries due to control surfaces deflections or fins misalignment is performed. Linear theory is mentioned in most of researches. In Vaughn (1968) and Eikensberry (1970) a detailed development of tricyclic theory is presented. Malmgrenproposed an approximate model for a rolling airframe having one pair control surface by averaging the force and moment equations in a nonrolling frame over one roll revolution (Malmgren, 1999). Lestage developed a modified dynamic model for rolling airframe with one-pair control surface and two-pair control surface (Lestage, 2000). He showed that the response of airframe controlled by one-pair is nearly equal to the response of airframe controlled by two-pairs but with half control effectiveness and additional disturbance on the commanded value modulated at twice the rolling frequency. The dynamic stability of rolling airframe actuated by DC motor is covered well in literature. For example, in Yan et al. (2010), the dynamic instability of rolling airframe caused by rate loop controller is studied and the suitable stability conditions are established. The effect of attitude and acceleration autopilots are discussed and the dynamic stability boundary is analytically derived (Yan et al., 2011; Li et al., 2012). The effects of DC motor actuator on the dynamic stability of rolling airframe is proposed in Zhou et al. (2013), Koohmaskan et al. (2015). Then, the sufficient conditions related to actuator's characteristics which keep the airframe in the safe region of dynamic stability are derived. A comparison between continuous and ON-OFF actuators is done by Koohmaskan et al. (2016). Nobahari et al. (2012) have utilized the multiple-input describing function technique for linearization the output of ON-OFF actuator. The performance of control and guidance system for a

*Corresponding author. E-mail: karimi_j@mut.ac.ir 
spinning flight vehicle with dithering canard is studied in Mirzaei and Alishahi (2014).

The effect of one pair ON-OFF control surface on dynamic stability behavior of a rolling airframe is concentrated in the current research. In this regard, a new analytical relation is derived for ideal and real ON-OFF control surface. Based on the linear theory, new closed form solutions are derived for rolling airframe motion in presence of ON-OFF control surface model. According to the author's knowledge, this is for the first time that such a formulation is proposed. Utilizing the new closed form solution, actuator model effect on rolling airframe dynamic behavior is evaluated. The resonance phenomenon, due to actuator dynamic model, is studied. The effect of rolling airframe roll rate magnitude on angular motion is also studied. The proposed closed form solution is validated by comparing its results with that of a numerical solution.

The paper is organized as follows: in section 1, four degree of freedom (4-DOF) equations of motion in nonrolling frame are proposed. In section 2 the analytical model for ideal and real actuator is illustrated. Section 3 is devoted to rolling airframe motion closed form solution derivation in presence of one pair ON-OFF control surface. Dynamic stability analysis based on proposed analytical solutions are performed in section 4 . Validation of proposed closed form solutions are shown in section 5 and conclusions are proposed in the last section.

\section{Airframe equations of motion}

Rolling airframe dynamic equations of motion are nonlinear six degree of freedom (Zipfel, 2007). However, because of dynamic behavior of rolling airframes, in a body fixed rolling frame, it can be assumed that $\mathrm{x}$-component of linear velocity in body frame $\left(V_{x}\right)$ and roll rate $(p)$ are constant. Hence, for stability analysis purposes, a linearized 4-DOF model including angle of attack $(\alpha)$, sideslip angle $(\beta)$, pitch rate $(q)$ and yaw rate $(r)$ is sufficient. Here, for canard-controlled rolling airframe with one pair control surface, it is assumed that that the $y$ component of the rolling frame is along with the control surface. Therefore, the control surface is supposed to be an elevator in rolling frame. It is more convenient to study dynamic stability in non-rolling frame with state vector $\mathbf{X}=\left[\beta_{\mathrm{NR}}, \alpha_{\mathrm{NR}}, q_{\mathrm{NR}}, r_{\mathrm{NR}}\right]^{\mathrm{T}}$. The relation between states in rolling and non-rolling frames is shown in Eq. (1).

$$
\left[\begin{array}{l}
\beta_{\mathrm{NR}} \\
\alpha_{\mathrm{NR}} \\
q_{\mathrm{NR}} \\
r_{\mathrm{NR}}
\end{array}\right]=\left[\begin{array}{cccc}
\cos (\varphi) & -\sin (\varphi) & 0 & 0 \\
\sin (\varphi) & \cos (\varphi) & 0 & 0 \\
0 & 0 & \cos (\varphi) & -\sin (\varphi) \\
0 & 0 & \sin (\varphi) & \cos (\varphi)
\end{array}\right]\left[\begin{array}{l}
\beta \\
\alpha \\
q \\
r
\end{array}\right] .
$$

Here, $\varphi$ is the angle between body-fixed rolling frame and non-rolling frame, as shown in Figure 1.

$$
\varphi(t)=\int_{0}^{t} p d t
$$

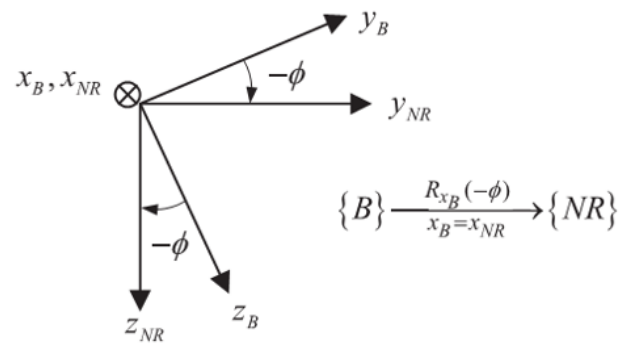

Figure 1. Transformation from rolling frame $\{B\}$ to non-rolling frame $\{\mathrm{NR}\}$

State space form of 4-DOF dynamic equation in nonrolling frame is written as follows (Mohammadi et al., 2016):

$\left[\begin{array}{c}\frac{d \beta_{\mathrm{NR}}}{d t} \\ \frac{d \alpha_{\mathrm{NR}}}{d t} \\ \frac{d q_{\mathrm{NR}}}{d t} \\ \frac{d r}{d t}\end{array}\right]=\left[\begin{array}{cccc}N_{\alpha} & 0 & 0 & -1 \\ 0 & N_{\alpha} & 1 & 0 \\ M_{p \alpha} & M_{\alpha} & M_{q} & -\frac{\mathrm{I}_{\mathrm{x}}}{\mathrm{I}_{\mathrm{y}}} p \\ -M_{\alpha} & M_{p \alpha} & \frac{\mathrm{I}_{\mathrm{x}}}{\mathrm{I}_{\mathrm{y}}} & M_{q}\end{array}\right]\left[\begin{array}{c}\beta_{\mathrm{NR}} \\ \alpha_{\mathrm{NR}} \\ q_{\mathrm{NR}} \\ r_{\mathrm{NR}}\end{array}\right]+\left[\begin{array}{c}-N_{\delta} \sin (\varphi) \\ N_{\delta} \cos (\varphi) \\ M_{\delta} \cos (\varphi) \\ M_{\delta} \sin (\varphi)\end{array}\right] \delta$,

where:

$$
\begin{aligned}
& N_{\alpha}=\frac{\rho V^{2} S C_{N \alpha}}{2 m u} ; \\
& M_{\alpha}=\frac{\rho V^{2} S d C_{m \alpha}}{2 I_{y}} ; \\
& M_{q}=\frac{\tilde{n} V^{2} S d C_{m q}}{2 \mathrm{I}_{\mathrm{y}}}\left(\frac{d}{2 V}\right) ; \\
& M_{p \alpha}=\frac{\tilde{n} V^{2} \mathrm{Sd} C_{m p \alpha}}{2 \mathrm{I}}\left(\frac{p d}{2 V}\right) \\
& N_{\delta}=\frac{\tilde{n} V^{2} \mathrm{~S} C_{N \delta}}{2 \mathrm{~m} u} ; \\
& M_{\delta}=\frac{\tilde{n} V^{2} \mathrm{Sd} C_{m \delta}}{2 \mathrm{I}_{\mathrm{y}}}
\end{aligned}
$$

Here, $\delta$ is the applied ON-OFF control command in rolling frame. Constants $\mathrm{m}, \mathrm{I}, \mathrm{S}$ and $\mathrm{d}$ are airframe mass, moment of inertia, reference area and reference length, respectively. $V$ is true air speed. Aerodynamic derivatives $C_{N \alpha}$ and $C_{N \delta}$ indicate variation of normal force with angle of attack and control surface. $C_{m \alpha}, C_{m q}$ and $C_{m \delta}$ are variation of pitching moment with angle of attack, pitch rate and elevator and finally, $C_{m p \alpha}$ is variation of pitching moment due to Magnus effect with angle of attack.

\section{Analytical model for ON-OFF control surface}

In current research, for stability analysis purpose, two mathematical models are used for the ON-OFF control 
surface, namely ideal and real models. In real model, the actuator is assumed to be zero-lag and hence, its transfer function is taken as 1 . Real actuator is assumed to be single-lag with transfer function $1 /(1+\tau S)$. Here, $\tau$ is set to be 0.005 second. Time history of ideal and real models, during two periods, is shown in Figure 2 and Figure 3.

The ideal ON-OFF control surface input can be represented by the following relation:

$$
\delta(t)=\left\{\begin{array}{ll}
+\delta_{\max } & \text { for } t \in\left[j T,\left(\frac{2 j+1}{2}\right) T\right] \\
-\delta_{\max } & \text { for } t \in\left[\left(\frac{2 j+1}{2}\right) T,(j+1) T\right]
\end{array},\right.
$$

where, $j=1,2, \ldots, N$ and $T$ is period of revolution $(T=2 \pi / p)$. The problem is that, Eq. (4) is not usable in analytical analysis, because it represents a discrete function. Therefore, in current research, a new analytical formulation is derived to handle this problem. In this way, from Eq. (4) and after some simplifications, the following relations can be derived for $N=1$ :

$$
\delta_{1}(t)=\delta_{\max }\left(u(t)-2 u\left(t-\frac{T}{2}\right)+u(t-T)\right) .
$$

Also, for $N=2$ it can be seen that:

$$
\begin{aligned}
& \delta_{2}(t)=\delta_{\max }\left(u(t)-2 u\left(t-\frac{T}{2}\right)+u(t-T)\right)+ \\
& \delta_{\max }\left(u(t-T)-2 u\left(t-\frac{3 T}{2}\right)+u(t-2 T)\right) .
\end{aligned}
$$

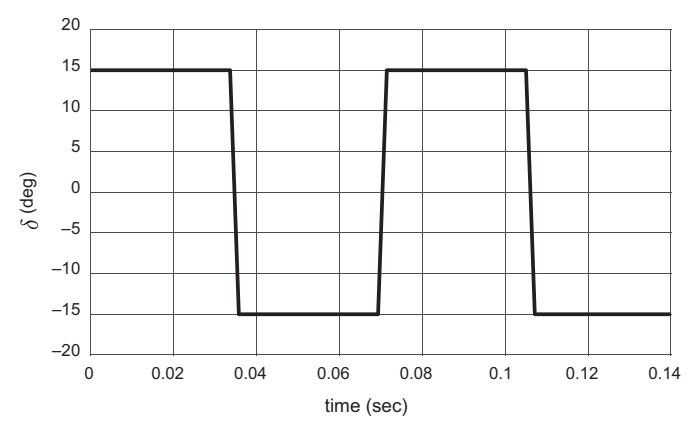

Figure 2. Time history of ideal ON-OFF actuator

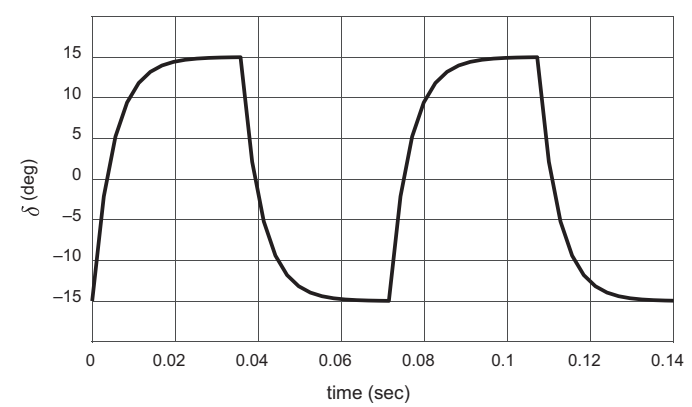

Figure 3. Time history of real ON-OFF actuator
Finally, utilizing mathematical induction theorem, the analytical formula of ideal ON-OFF control surface during $N$ periods is given as

$$
\begin{aligned}
& \delta_{N}(t)=\delta_{\max } \sum_{j=1}^{N-1}\left(u(t-j T)-u\left(t-\frac{2 j+1}{2} T\right)\right)- \\
& \delta_{\max } \sum_{j=1}^{N}\left(u\left(t-\frac{2 j-1}{2} T\right)-u(t-j T)\right) .
\end{aligned}
$$

Here, $u(t)$ is unit step function. In a similar way to the ideal model, for real ON-OFF control surface in the case of $N=1$ and $N=2$ the following relations can be derived:

$$
\begin{aligned}
& \delta_{1}(\mathrm{t})=\delta_{\max }\left(1-2 e^{-\frac{t}{\tau}}\left(u(t)-u\left(t-\frac{T}{2}\right)\right)\right)- \\
& 2 \delta_{\max }\left(\left(1-e^{-\frac{t-\frac{T}{2}}{\tau}}\right)\left(u\left(t-\frac{T}{2}\right)-u(t-T)\right) ;\right. \\
& \delta_{2}(t)=\delta_{\max }\left(1-2 e^{-\frac{t}{\tau}}\left(u(t)-u\left(t-\frac{T}{2}\right)\right)\right)- \\
& \delta_{\max }\left(2 e^{-\frac{t-T}{\tau}}\left(u(t-T)-u\left(t-\frac{3 T}{2}\right)\right)\right) \\
& -2 \delta_{\max }\left(1-e^{-\frac{1}{\tau}\left(t-\frac{T}{2}\right)}\right)\left(u\left(t-\frac{T}{2}\right)-u(t-T)\right)- \\
& 2 \delta_{\max }\left(1-e^{-\frac{1}{\tau}\left(t-\frac{3 T}{2}\right)}\right)\left(u\left(t-\frac{3 T}{2}\right)-u(t-2 T)\right) .
\end{aligned}
$$

Again, by mathematical induction theorem, for $N$ periods, the analytical formulation of real ON-OFF control surface input command is given as

$$
\begin{aligned}
& \delta_{N}(t)=\delta_{\max }-2 \delta_{\max } \sum_{j=0}^{N-1} e^{-\frac{t-j T}{\tau}}\left(u(t-j T)-u\left(t-\frac{2 j+1}{2} T\right)\right)- \\
& 2 \delta_{\max } \sum_{j=1}^{N}\left(1-e^{-\frac{t-\frac{2 j-1}{2} T}{\tau}}\right)\left(u\left(t-\frac{2 j-1}{2} T\right)-u(t-j T)\right) .
\end{aligned}
$$

\section{Closed form solution derivation}

By introducing the following two complex variables

$$
\begin{aligned}
& \xi=\beta_{\mathrm{NR}}+i \alpha_{\mathrm{NR}} ; \\
& \mu=q_{\mathrm{NR}}+i r_{\mathrm{NR}} .
\end{aligned}
$$
1963)

Eq. (3) takes the following simplified form (Murphy,

$$
\frac{d \xi}{d t}=N_{\alpha} \xi+i \mu+i N_{\delta} \delta_{N}(t) e^{i p t}
$$




$$
\frac{d \mu}{d t}=M_{p \alpha} \xi-i M_{\alpha} \xi+i I_{p} \mu+M_{q} \mu+M_{\delta} \delta_{N}(t) e^{i p t}
$$

Omitting variable $\mu$ from Eq. (13) and Eq. (14), leads to a non-homogeneous second-order complex differential equation with discontinuous forcing term.

$$
\frac{d^{2} \xi}{d t^{2}}-\left(H+i I_{p}\right) \frac{d \xi}{d t}+\left(M+i I_{p} T_{p}\right) \xi=i M_{c} \delta_{N}(t) e^{i p t}
$$

$$
\begin{aligned}
& \text { where, } \\
& H=M_{q}+N_{\alpha}, I_{p}=\left(\frac{I_{x}}{I_{y}}\right) p, M=M_{q} N_{\alpha}-M_{\alpha}, \\
& T_{p}=N_{\alpha}-\frac{M_{p \alpha}}{I_{p}}, M_{c}=M_{\delta}-M_{q} N_{\delta}+i N_{\delta} p-i I_{p} N_{\delta} .
\end{aligned}
$$

Variables $H, I_{p}, M, T_{p}$ and $M_{c}$ represent effects of aerodynamic damping, gyroscopic motion, aerodynamic stability, Magnus moment and control surface, respectively (Zhou et al., 2014).

Before studying the effects of ON-OFF control surface on airframe's dynamic stability, a brief overview of airframe's stability in free flight conditions will be presented. Assuming the control signal is zero $\left(\delta_{N}(t)=0\right)$, the solution of homogeneous form for Eq. (15) is an epicyclical motion including a low frequency motion called precession and a high frequency motion namely nutation (Vaughn, 1968; Eikensberry, 1970):

$$
\xi=K_{1} e^{\phi_{1} t}+K_{2} e^{\phi_{2} t}
$$

$\phi_{1}=\lambda_{1}-i \omega_{1}=\frac{\left(H+i I_{p}\right)+\sqrt{\left(H+i I_{p}\right)^{2}-4\left(M+i I_{p} T_{p}\right)}}{2} ;$

$$
\phi_{2}=\lambda_{2}+i \omega_{2}=\frac{\left(H+i I_{p}\right)-\sqrt{\left(H+i I_{p}\right)^{2}-4\left(M+i I_{p} T_{p}\right)}}{2}
$$

$$
\begin{gathered}
K_{1}=\frac{\frac{d \xi_{0}}{d t}-\phi_{2} \xi_{0}}{\phi_{1}-\phi_{2}} \\
K_{2}=\frac{\frac{d \xi}{d t}-\phi_{1} \xi_{0}}{\phi_{2}-\phi_{1}} .
\end{gathered}
$$

First term of Eq. (16) represents precession and second term is for nutation. The motion amplitude $\left(K_{1,2}\right)$ can either grow or decrease, due to initial conditions, while the frequency $\left(\omega_{1,2}\right)$ is constant. In statically stable airframes, the shape of airframe response in $\alpha_{\mathrm{NR}}-\beta_{\mathrm{NR}}$ plane, is given by slowly rotating ellipses for small roll rates, flowerlike for medium roll rates, and for large roll rates is similar to the fast spinning gyroscopic pendulum ${ }^{1}$.

If a constant control surface or an asymmetric term is taken into account, $\left(\delta_{N}(t)=\delta\right)$, the solution of generated non-homogeneous form of Eq. (15) is called tricyclic mo- tion including precession, nutation and constant control surface effect (Nicolaides, 1953):

$$
\xi=K_{1} e^{\phi_{1} t}+K_{2} e^{\phi_{2} t}+K_{3} e^{i p t},
$$

where,

$$
\begin{aligned}
& K_{1}=\frac{\frac{d \xi_{0}}{d t}-\phi_{2} \xi_{0}+K_{3}\left(\phi_{2}-\phi_{3}\right)}{\phi_{1}-\phi_{2}} \\
& K_{2}=\frac{\frac{d \xi_{0}}{d t}-\phi_{1} \xi_{0}+K_{3}\left(\phi_{1}-\phi_{3}\right)}{\phi_{2}-\phi_{1}} ; \\
& K_{3}=\frac{i M_{c} \delta}{\left(\phi_{1}-i p\right)\left(\phi_{2}-i p\right)} .
\end{aligned}
$$

The third term of Eq. (21), not only affects amplitude of nutation and precession modes via changing $K_{1}$ and $K_{2}$ terms, in comparison to symmetrical case, but also may cause the "resonance instability phenomenon". According to Eq. (24), if the denominator of third term converges to zero, infinite amplitudes can be reached and resonance instability takes place. It can be shown that in statically stable airframes, resonance instability could take place for nutation mode, i.e. airframe's roll rate becomes equal or near to nutation frequency. In practice, occurrence of resonance instability in precession mode is impossible.

The dynamic stability of rolling airframe, in both free flight case and in presence of constant control surface, is widely studied in literature. But the effect of ON-OFF control input on rolling airframe dynamic stability isn't presented previously. In this way, a new closed form solution problem will be derived. Taking Laplace transform of Eq. (15) leads to:

$$
\begin{aligned}
& S^{2} \xi(S)-S \xi_{0}-\frac{d \xi_{0}}{d t}-\left(H+i I_{p}\right)\left(S \xi(S)-\xi_{0}\right)+ \\
& \left(M+i I_{p} T_{p}\right) \xi(S)=i M_{c} \mathcal{L}\left(\delta_{N}(t) e^{i p t}\right),
\end{aligned}
$$

where $\mathcal{L}$ indicates the Laplace transformation. Eq. (25) could be rearranged as below:

$$
\begin{gathered}
\xi(S)=\frac{S \xi_{0}+\frac{d \xi_{0}}{d t}-\left(H+i I_{p}\right) \xi_{0}}{S^{2}-\left(H+i I_{p}\right) S+\left(M+i I_{p} T\right)}+ \\
\frac{i M_{c} \mathcal{L}\left(\delta_{N}(t) e^{i p t}\right)}{S^{2}-\left(H+i I_{p}\right) S+\left(M+i I_{p} T\right)} .
\end{gathered}
$$

Utilizing Laplace transformation method (Dawkins, 2011) and previously generated models for ideal and real ON-OFF control surfaces, see section 3, new closed form solutions for Eq. (26) are derived.

\subsection{Closed form solution for ideal ON-OFF control surface}

Let us substitute the analytical model of ideal ON-OFF control surface, Eq. (7), into rolling airframe dynamic 
model in frequency domain, Eq. (26) and get Laplace transformation from resulted relation. After needed complex mathematical calculations, the following closed form solution is derived in frequency domain:

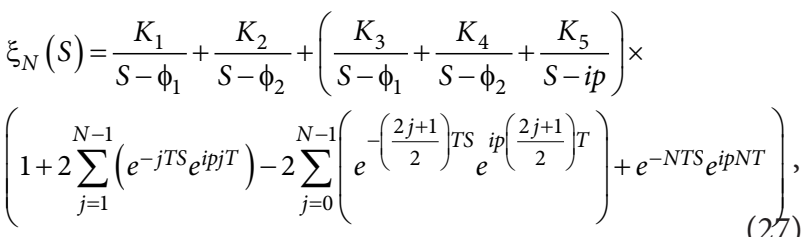

where,

$$
\begin{aligned}
& K_{1}=\frac{\frac{d \xi_{0}}{d t}+\phi_{1} \xi_{0}-\left(H+i I_{p}\right) \xi_{0}}{\phi_{1}-\phi_{2}}=\frac{\frac{d \xi_{0}}{d t}-\phi_{2} \xi_{0}}{\phi_{1}-\phi_{2}} ; \\
& K_{2}=\frac{\frac{d \xi_{0}}{d t}+\phi_{2} \xi_{0}-\left(H+i I_{p}\right) \xi_{0}}{\phi_{2}-\phi_{1}}=\frac{\frac{d \xi_{0}}{d t}-\phi_{1} \xi_{0}}{\phi_{2}-\phi_{1}} ; \\
& K_{3}=\frac{i M_{c} \delta_{\max }}{\left(\phi_{1}-\phi_{2}\right)\left(\phi_{1}-i p\right)} ; \\
& K_{4}=\frac{i M_{c} \delta_{\max }}{\left(\phi_{2}-\phi_{1}\right)\left(\phi_{2}-i p\right)} ; \\
& K_{5}=\frac{i M_{c} \delta_{\max }}{\left(i p-\phi_{1}\right)\left(i p-\phi_{2}\right)} .
\end{aligned}
$$

Variables $\phi_{1}$ and $\phi_{2}$ are denominator roots of Eq. (26) and previously obtained in Eq. (17) and Eq. (18). Proof of Eq. (27) is presented in Appendix A.

By taking inverse Laplace transformation of Eq. (26) the following closed form solution is developed in time domain:

$$
\xi_{N}(t)=K_{1} e^{\phi_{1} t}+K_{2} e^{\phi_{2} t}+\xi_{\phi_{1}}+\xi_{\phi_{2}}+\xi_{p}
$$

where,

$$
\begin{aligned}
& \frac{\xi_{\phi_{1}}}{K_{3}}=e^{\phi_{1} t}+2 \sum_{j=1}^{N-1}\left(e^{\phi_{1}(t-j T)} e^{i p j T} u(t-j T)\right)+e^{\phi_{1}(t-N T)} e^{i p N T} u(t-N T)- \\
& 2 \sum_{j=0}^{N-1}\left(e^{\phi_{1}\left(t-\frac{2 j+1}{2} T\right)} e^{i p \frac{2 j+1}{2} T} u\left(t-\frac{2 j+1}{2} T\right)\right) \text {; }
\end{aligned}
$$

$\frac{\xi_{\phi_{2}}}{K_{4}}=e^{\phi_{2} t}+2 \sum_{j=1}^{N-1} e^{\phi_{2}(t-j T)} e^{i p j T} u(t-j T)+e^{\phi_{2}(t-N T)} e^{i p N T} u(t-N T)-$

$2 \sum_{j=0}^{N-1} e^{\phi_{2}\left(t-\frac{2 j+1}{2} T\right)} e^{i p \frac{2 j+1}{2} T} u\left(t-\frac{2 j+1}{2} T\right) ;$

$\frac{\xi_{p}}{K_{5}}=e^{i p t}+2 \sum_{j=1}^{N-1} e^{i p t} u(t-j T)+e^{i p t} u(t-N T)-$

$2 \sum_{j=0}^{N-1} e^{i p t} u\left(t-\frac{2 j+1}{2} T\right)$

The solution has five terms. From Eq. (34), Eq. (35) and Eq. (36) it can be seen that ON-OFF control affects both amplitude and phase of nutation and precession modes. Another notable result reveals from this analyti- cal solution is that in the case of ON-OFF control surface, relating to $K_{4}$ and $K_{5}$ terms, resonance instability may take place. Substituting $\phi_{1,2}$ from Eq. (17) and Eq. (18) into denominator of Eq. (31) and Eq. (32) and making some simplifications results in following parametric relation for critical roll rate at which resonance takes place:

$$
p_{\text {resonance }}=\frac{I_{p}}{2}+\sqrt{\frac{I_{p}^{2}}{4}+M} \text {. }
$$

\subsection{Closed form solution for real ON-OFF control surface}

In a similar way to ideal case, substituting Eq. (10) in Eq. (26) and using Laplace transformation properties, a new solution is derived in frequency domain, for rolling airframe dynamic with real ON-OFF control surface:

$$
\begin{aligned}
& \xi_{N}(S)=\frac{K_{1}}{S-\phi_{1}}+\frac{K_{2}}{S-\phi_{2}}+\left(\frac{K_{3}}{S-\phi_{1}}+\frac{K_{4}}{S-\phi_{2}}+\frac{K_{5}}{S-i p+\frac{1}{\tau}}\right) \times \\
& \left(-2 \sum_{j=1}^{N} e^{-(j-1) T S} e^{i p(j-1) T}+2 \sum_{j=1}^{N} e^{-\frac{2 j-1}{2} T S} e^{i p \frac{2 j-1}{2} T}\left(1+e^{\frac{-T}{2 \tau}}\right)-2 \sum_{j=1}^{N} e^{-j T S} e^{i p j T-\frac{T}{2 \tau}}\right)+ \\
& \left(\frac{k_{33}}{S-\phi_{1}}+\frac{k_{44}}{S-\phi_{2}}+\frac{k_{55}}{S-i p}\right)\left(1-2 \sum_{j=1}^{N} e^{-\frac{2 j-1}{2} T S} e^{i p \frac{2 j-1}{2} T}+2 \sum_{j=1}^{N} e^{-j T S} e^{i p j T}\right) .
\end{aligned}
$$

Variables $K_{1}$ and $K_{2}$ are similar to that of ideal case, Eq. (28) and Eq. (29) and the other new terms are defined as below:

$$
\begin{aligned}
& K_{3}=\frac{i M_{c} \delta_{\max }}{\left(\phi_{1}-\phi_{2}\right)\left(\phi_{1}-i p+\frac{1}{\tau}\right)} ; \\
& K_{4}=\frac{i M_{c} \delta_{\max }}{\left(\phi_{2}-\phi_{1}\right)\left(\phi_{2}-i p+\frac{1}{\tau}\right)} ; \\
& K_{5}=\frac{i M_{c} \delta_{\max }}{\left(\phi_{1}-i p+\frac{1}{\tau}\right)\left(\phi_{2}-i p+\frac{1}{\tau}\right)} ; \\
& K_{33}=\frac{i M_{c} \delta_{\max }}{\left(\phi_{1}-\phi_{2}\right)\left(\phi_{1}-i p\right)} ; \\
& K_{44}=\frac{i M_{c} \delta_{\max }}{\left(\phi_{2}-\phi_{1}\right)\left(\phi_{2}-i p\right)} ; \\
& K_{55}=\frac{i M_{c} \delta_{\max }}{\left(\phi_{1}-i p\right)\left(\phi_{2}-i p\right)} .
\end{aligned}
$$

Appendix B presents the proof of Eq. (38). Now, by taking inverse Laplace transformation of Eq. (38) the complex angle of attack , in case of real control command will be given as bellow:

$$
\xi_{N}(t)=K_{1} e^{\phi_{1} t}+K_{2} e^{\phi_{2} t}+\xi_{\phi_{1}}+\xi_{\phi_{2}}+\xi_{p},
$$


where,

$$
\begin{aligned}
& \xi_{\phi_{1}}=K_{33} e^{\phi_{1} t}-2 K_{3} \sum_{j=1}^{N} e^{\phi_{1}(t-(j-1) T)} e^{i p(j-1) T} u(t-(j-1) T)+ \\
& 2 K_{3} \sum_{j=1}^{N} e^{\phi_{1}\left(t-\frac{2 j-1}{2} T\right)} e^{i p \frac{2 j-1}{2} T}\left(1+e^{\frac{-T}{2 \tau}}\right) u\left(t-\frac{2 j-1}{2} T\right)- \\
& 2 K_{3} \sum_{j=1}^{N} e^{\phi_{1}(t-j T)} e^{i p j T-\frac{T}{2 \tau}} u(t-j T)- \\
& 2 K_{33} \sum_{j=1}^{N} e^{\phi_{1}\left(t-\frac{2 j-1}{2} T\right)} e^{i p \frac{2 j-1}{2} T} u\left(t-\frac{2 j-1}{2} T\right)+ \\
& 2 K_{33} \sum_{j=1}^{N} e^{\phi_{1}(t-j T)} e^{i p j T-\frac{T}{2 \tau}} u(t-j T) ; \\
& \xi_{\phi_{2}}=K_{44} e^{\phi_{2} t}-2 K_{4} \sum_{j=1}^{N} e^{\phi_{2}(t-(j-1) T)} e^{i p(j-1) T} u(t-(j-1) T)+ \\
& 2 K_{4} \sum_{j=1}^{N} e^{\phi_{2}\left(t-\frac{2 j-1}{2} T\right)} e^{i p^{2 j-1}} \frac{2 j}{2}\left(1+e^{\frac{-T}{2 \tau}}\right) u\left(t-\frac{2 j-1}{2} T\right)- \\
& 2 K_{4} \sum_{j=1}^{N} e^{\phi_{2}(t-j T)} e^{i p j T-\frac{T}{2 \tau}} u(t-j T)_{-} \\
& 2 K_{44} \sum_{j=1}^{N} e^{\phi_{2}\left(t-\frac{2 j-1}{2} T\right)} e^{i p \frac{2 j-1}{2} T} u\left(t-\frac{2 j-1}{2} T\right)+ \\
& 2 K_{44} \sum_{j=1}^{N} e^{\phi_{2}(t-j T)} e^{i p j T-\frac{T}{2 \tau}} u(t-j T) ; \\
& \xi_{p}=K_{55} e^{i p t}-2 K_{5} \sum_{j=1}^{N} e^{\left(i p-\frac{1}{\tau}\right)(t-(j-1) T)} e^{i p(j-1) T} u(t-(j-1) T)+ \\
& 2 K_{5} \sum_{j=1}^{N} e^{\left(i p-\frac{1}{\tau}\right)\left(t-\frac{2 j-1}{2} T\right)} e^{i p \frac{2 j-1}{2} T}\left(1+e^{\frac{-T}{2 \tau}}\right) u\left(t-\frac{2 j-1}{2} T\right)- \\
& 2 K_{5} \sum_{j=1}^{N} e^{\left(i p-\frac{1}{\tau}\right)(t-j T)} e^{i p j T-\frac{T}{2 \tau}} u(t-j T)_{-} \\
& 2 K_{55} \sum_{j=1}^{N} e^{i p t} u\left(t-\frac{2 j-1}{2} T\right)+2 K_{55} \sum_{j=1}^{N} e^{i p t-\frac{T}{2 \tau}} u(t-j T) .
\end{aligned}
$$

Eq. (45) represents a new analytical formula for angular motion of airframe in presence of real ON-OFF control model. In comparison to ideal case, it can be concluded that the resonance also takes place in real case. This can be seen in $K_{44}$ and $K_{55}$ terms. Existence of time delay term $\tau$ avoids the resonance in $K_{4}$ and $K_{5}$. Considering the time delay of actuator, it shifts the angular motion from that of ideal case, this can be seen from $e^{\frac{-T}{2 \tau}}$ term in $\xi_{\phi_{1}}, \xi_{\phi 2}$ and $\xi_{p}$.

\section{Dynamic stability analysis}

In this section, the stability of ON-OFF canard controlled rolling airframe is proposed through simulation. The effect of ON-OFF control terms on airframe's angular motion is also presented.

\subsection{Angular motion of ON-OFF controlled airframe}

Using numerical values of Table 1, and proposed closed form solutions, the planar and 3-dimensional responses with ideal and real ON-OFF controls are drawn in Figure 4 and Figure 5. The initial conditions are selected as $\xi_{0}=\beta_{\mathrm{NR} 0}+i \alpha_{\mathrm{NR} 0}=3+2 i$ and $\frac{d \xi_{0}}{d t}=\frac{d \beta_{\mathrm{NR} 0}}{d t}+\frac{d \alpha_{\mathrm{NR} 0}}{d t} i=0+0 i$. As can be seen, the angular motion forms a limited cycle. This shows a dynamically stable behavior. This result is compatible with that of (Mohammadi et al., 2016). It can also be seen that the angular motion in real actuator is shifted down, in comparison with ideal actuator. This is due to the single lag dynamic model of actuator which plays a role of time shift. And finally one can say that in steady state condition, the diameter of limit cycles in real actuator is smaller than ideal actuator. This is related to the fact that the average control effectiveness in presence of actuator's dynamic model is less than ideal actuator.

Table 1. Parameters of a rolling airframe

\begin{tabular}{|c|c|}
\hline Parameter & Value \\
\hline $\mathrm{H}$ & -16.696 \\
\hline $\mathrm{M}$ & 1897.042 \\
\hline $\mathrm{I}_{\mathrm{p}}$ & $0.431 \mathrm{rad} / \mathrm{sec}$ \\
\hline $\mathrm{T}$ & 15.429 \\
\hline $\mathrm{M}_{\mathrm{c}}$ & $1399.304+25.01 i$ \\
\hline$\delta_{\max }$ & $15 \mathrm{deg}$ \\
\hline $\mathrm{P}$ & $15 \mathrm{~Hz}$ \\
\hline
\end{tabular}

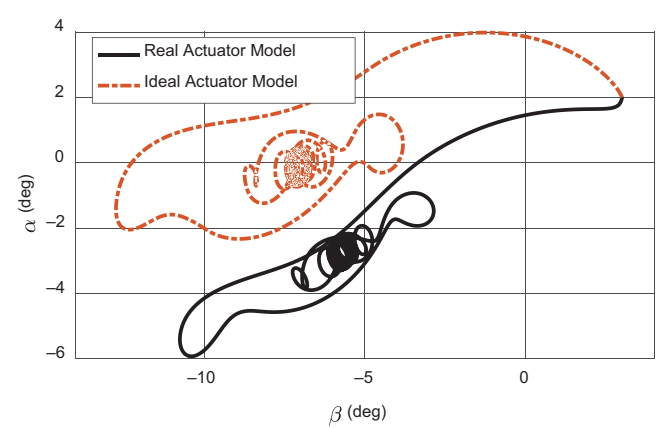

Figure 4. Rolling airframe planner motion representation in presence of real and ideal ON-OFF actuator models

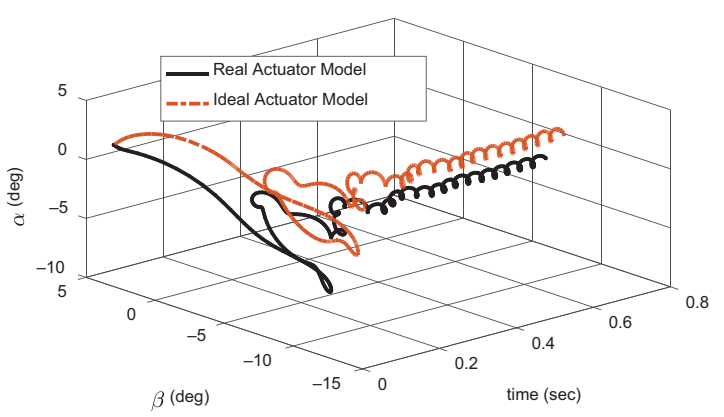

Figure 5. Three-dimensional angular motion in presence of real and ideal ON-OFF actuator model 


\subsection{Effect of roll rate on resonance instability}

In section 3 , it was analytically proven that the ON-OFF actuator affects both amplitude and phase of nutation and precession motions. We also stated that both $\xi_{\phi 2}$ and $\xi_{p}$ terms may cause resonance instability. Now, utilizing obtained closed form solutions and numerical values of Table 1, several analyses are down. It is clear that each term of Eq. (33) and Eq. (44) is a complex number with real and imaginary parts. Here, Euclidian norm of each term is called amplitude of that term. In literature this is called total angle of attack. Amplitude of $\xi_{\phi 1}, \xi_{\phi 2}$ and $\xi_{p}$ terms, for ideal actuator, at a constant roll rate $p=15 \mathrm{~Hz}$ are drawn in Figure 6, Figure 7 and Figure 8. The oscillatory behavior of these terms is because of discontinuous ON-OFF actuator. Also it can be seen that all three terms converge to a limited cycle. This states that in the case of nominal roll rate $p=15 \mathrm{~Hz}$ there is no evidence of dynamic instability.

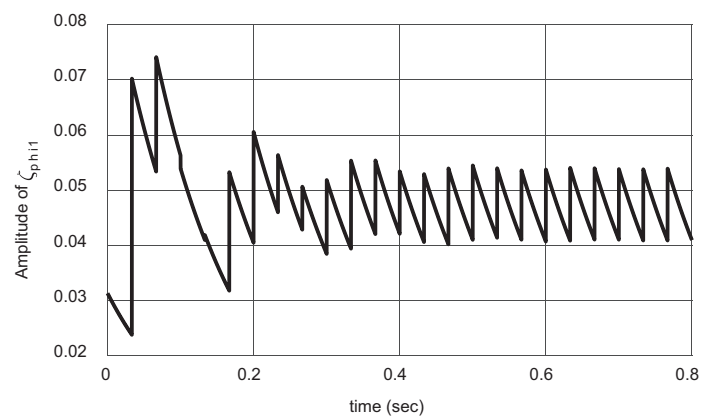

Figure 6. Time history of $\xi_{\phi 1}$ amplitude in ideal ON-OFF actuator for $p=15 \mathrm{~Hz}$

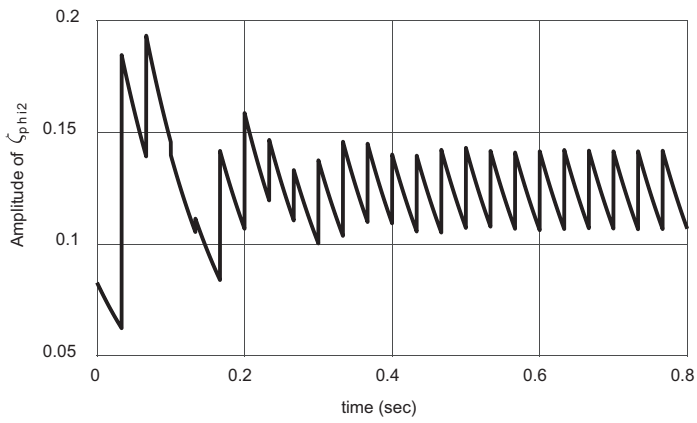

Figure 7. Time history of $\xi_{\phi 2}$ amplitude in ideal ON-OFF actuator for $p=15 \mathrm{~Hz}$

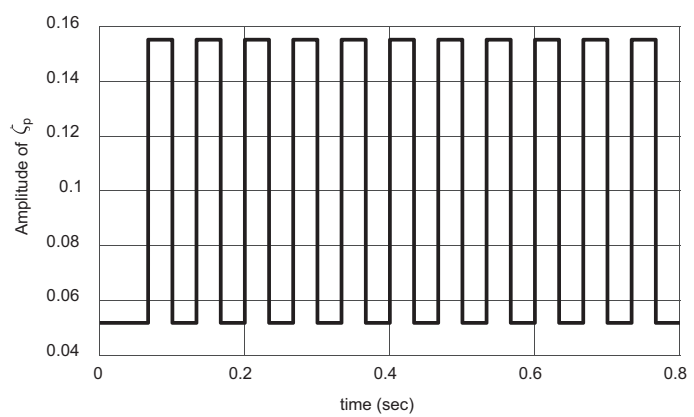

Figure 8. Time history of $\xi_{p}$ amplitude in ideal ON-OFF actuator for $p=15 \mathrm{~Hz}$
Now, the effect of rolling airframe roll rate on dynamic stability behavior with ideal actuator is studied in Figure 9, Figure 10 and Figure 11. As shown, both $\xi_{\phi 2}$ and $\xi_{p}$ reach the maximum amplitude when roll rate converges to nutation frequency, i.e. $\omega_{2}=42.96 \mathrm{rad} / \mathrm{sec}$. While, $\xi_{\phi 1}$ reaches its maximum amplitude at frequency $22 \mathrm{rad} / \mathrm{sec}$. This value is not possible to be reached and $\xi_{\phi 1}$ doesn't cause any resonance. Considering the rolling airframe roll rate range, one can judge about the occurrence of resonance phenomena arising from both $\xi_{\phi 2}$ and $\xi_{p}$ terms.

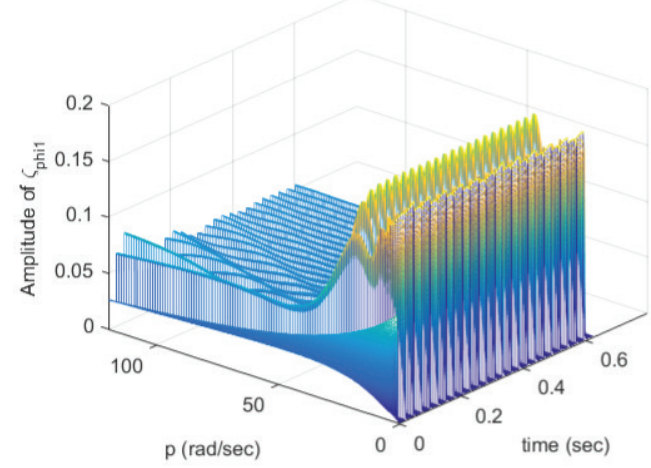

Figure 9. Roll rate effect on time history of $\xi_{\phi 1}$ amplitude in ideal ON-OFF actuator

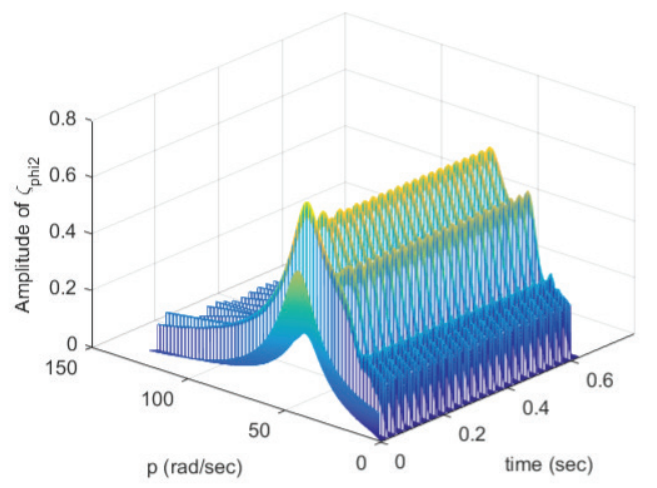

Figure 10. Roll rate effect on time history of $\xi_{\phi 2}$ amplitude in ideal ON-OFF actuator

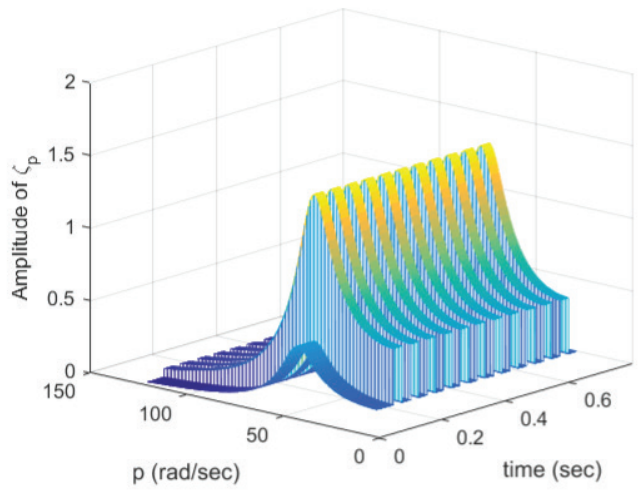

Figure 11. Roll rate effect on time history of $\xi_{p}$ amplitude in ideal ON-OFF actuator 
Similarly, for real ON-OFF control command, amplitudes of $\xi_{\phi 1}, \xi_{\phi 2}$ and $\xi_{p}$ terms with respect to time and airframe roll rate are drawn in Figure 12, Figure 13 and Figure 14. It can be seen that the amplitude of $\xi_{p}$ in real ON-OFF control is smaller rather than that of ideal control. It also can observed that these three control terms, after passing their peak value, tend to decrease with the roll rate growth.

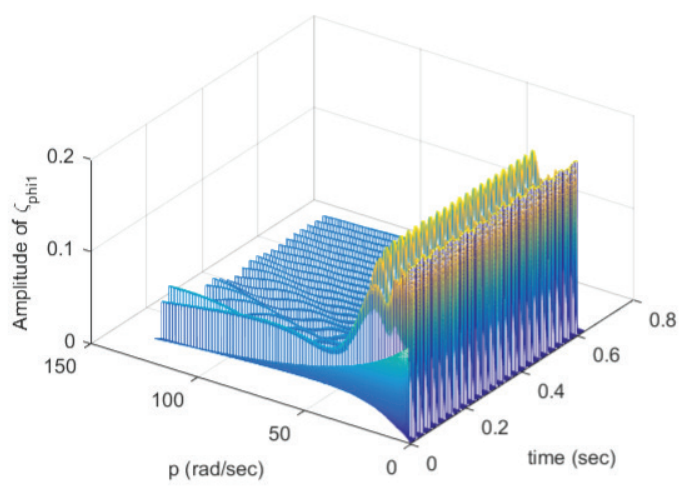

Figure 12. Roll rate effect on time history of $\xi_{\phi 1}$ amplitude in real $\mathrm{ON}-\mathrm{OFF}$ actuator

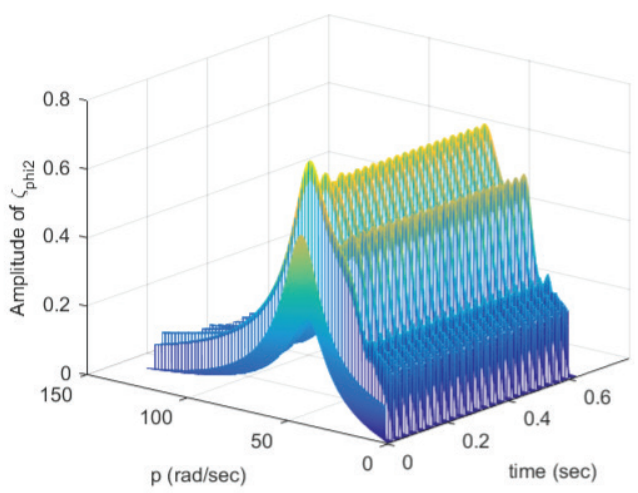

Figure 13. Roll rate effect on time history of $\xi_{\phi 2}$ amplitude in real ON-OFF actuator

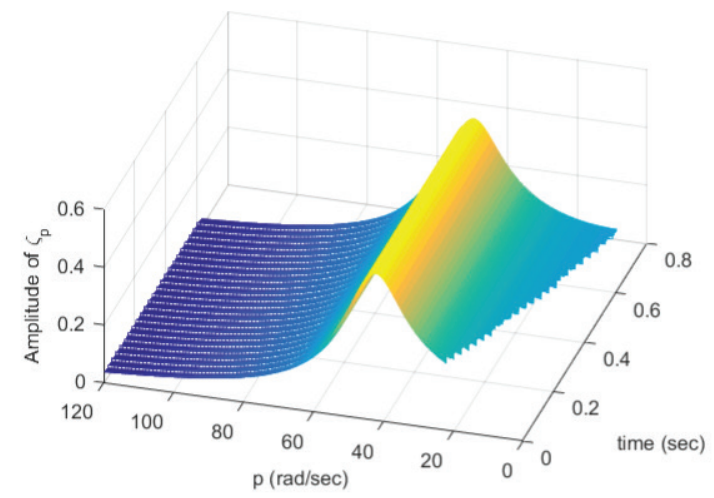

Figure 14. Roll rate effect on time history of $\xi_{p}$ amplitude in real ON-OFF actuator
From previous figures, in both ideal and real ON-OFF control commands, it can be seen that roll rate doesn't cause divergence situation, but it might cause critical dynamic stability in which the diameter of limit cycle increases largely. The limit cycle reach's the maximum diameter when roll rate converges to nutation frequency $p=42.96 \mathrm{rad} / \mathrm{sec} \approx 6.8 \mathrm{~Hz}$. As a result, to keep rolling airframe in safe region of dynamic stability, the roll rate should be bigger than twice nutation frequency. The difference of diameter in formed limit cycle between critical and nominal roll rate is shown in Figure 15 and Figure 16 for both ideal and real ON-OFF actuator respectively.

At last, the dynamic response is drawn for two different roll rates, in both ideal and real controls in Figure 17 and Figure 18. These figures clarify that, in steady state conditions, the diameter of limit cycle when $P=20 \mathrm{~Hz}$ is smaller than that of $P=15 \mathrm{~Hz}$. Therefore, one can say that in steady state conditions, the control terms are effective on the angular motion behavior. The total dynamic motion amplitude decreases when roll rate increases, due to amplitude descending trend of three control terms $\xi_{\phi 1}, \xi_{\phi 2}$ and $\xi_{p}$ with roll rate growth. Finally, from Figure 18, it can be seen that in presence of actuator dynamic, increasing roll rate causes the angular motion to be shifted down.

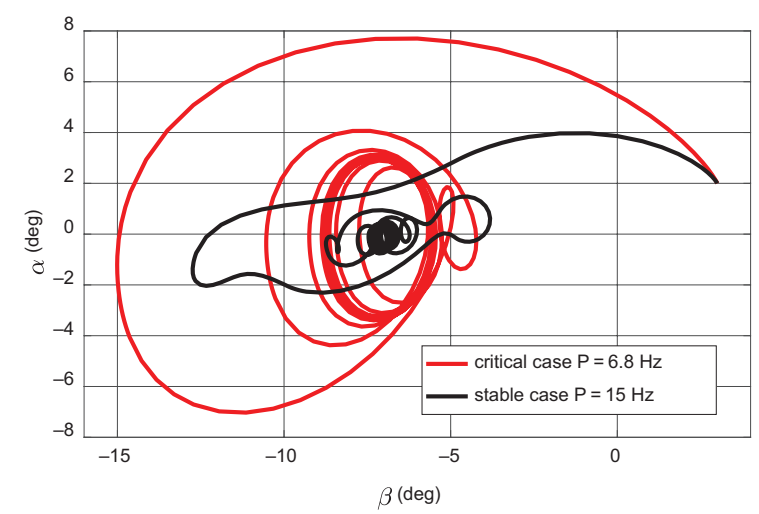

Figure 15. Diameter of limit cycle in critical and nominal roll rate for ideal ON-OFF actuator

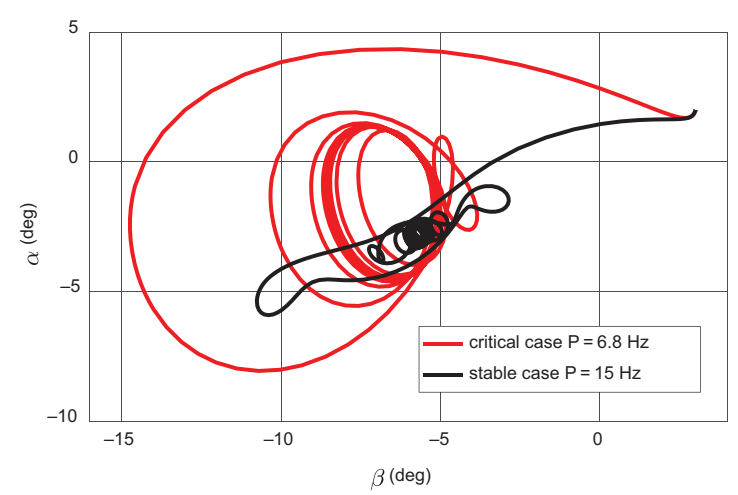

Figure 16. Diameter of limit cycle in critical and nominal roll rate for real $\mathrm{ON}-\mathrm{OFF}$ actuator 


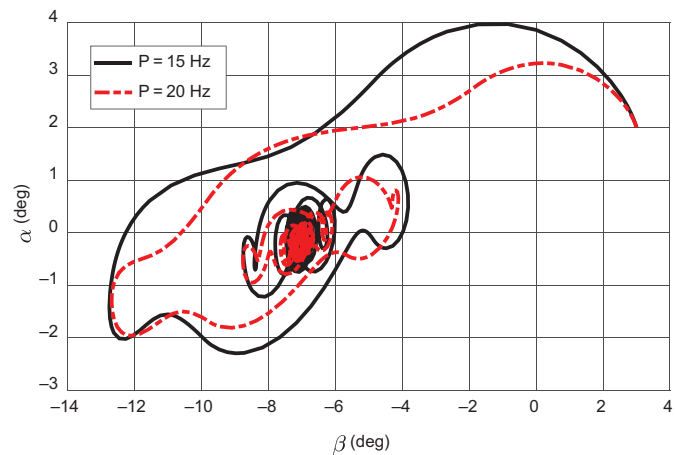

Figure 17. Effect of roll rate changing on ideal ON-OFF controlled rolling airframe

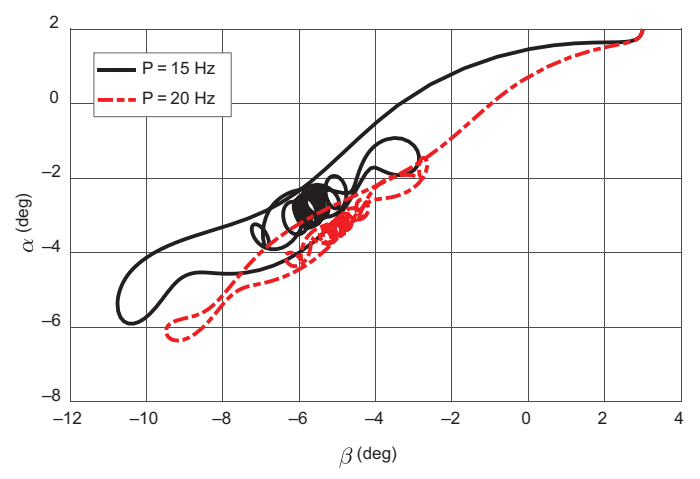

Figure 18. Effect of roll rate changing on real ON-OFF controlled rolling airframe

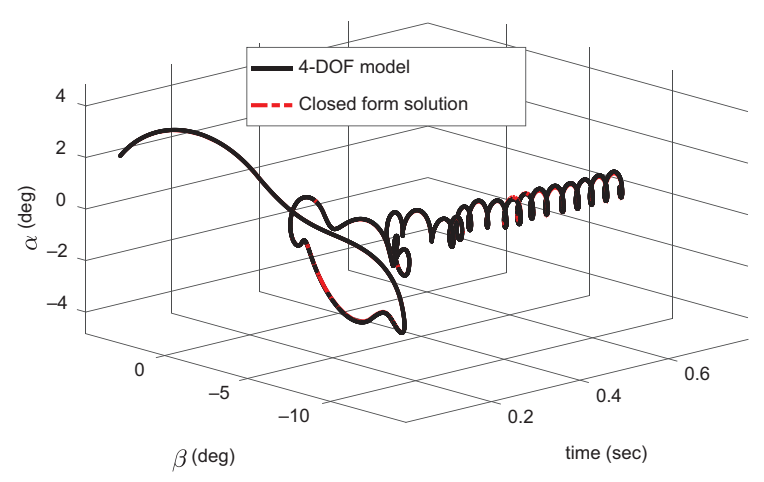

Figure 19. Comparison between closed form solution and linear 4-DOF model with ideal ON-OFF control

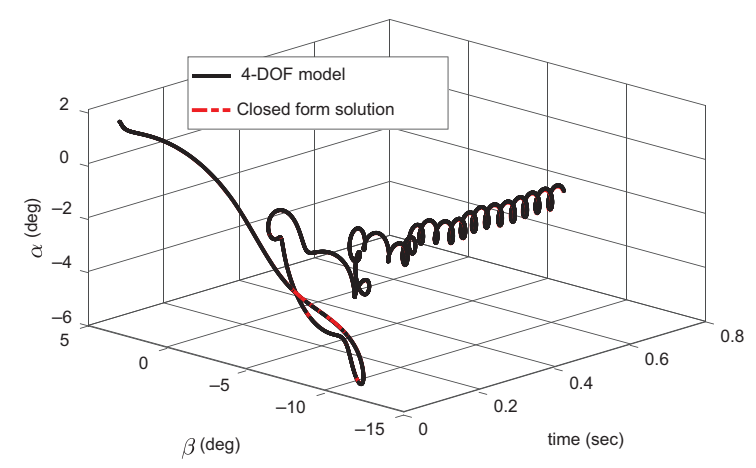

Figure 20. Comparison between closed form solution and linear 4-DOF model with real ON-OFF control

\section{Validation of proposed closed form solutions}

In this section, the new closed form solutions are compared with linear 4-DOF equations of motion, Eq. (3), in Figure 19 and Figure 20. 4-DoF equations are solved utilizing ode45 solver of MATLAB software. Ode45 is a numerical integration method based on an explicit RungeKutta $(4,5)$ formula, the Dormand- Prince pair (Shampine \& Reichelt, 1997; Dormand \& Prince, 1980). And the comparison was done for the same parameters shown in Table 1 and for that initial conditions used in section 4.1. As can be seen, the closed form solutions in both ideal ON-OFF control and real ON-OFF control provide dynamic behavior similar to original 4-DOF model.

\section{Conclusions}

In this paper, the linear theory is used in studying the dynamic stability of rolling airframe with one pair ON-OFF actuator. New closed form solutions are developed for ideal and real one pair ON-OF control. Utilizing the developed analytical models, the following notable results are obtained: It is shown that the ON-OFF control affects both amplitude and phase of nutation and precession motions. The resonance resources are determined and the effect of roll rate on the amplitudes of added ON-OFF control terms is studied. It is found that, when the actuator dynamic is taken in consideration (in real case), the angular motion is shifted from that in ideal case. This is due to the dynamic of actuator which plays a role of time shift. It was also found that in steady state conditions, the diameter of limit cycles in real case is smaller than that of ideal case. This is related to the fact that the average control effectiveness in the presence of actuator's dynamic is less than that in case of neglecting actuator dynamic. Finally, it is illustrated that increasing the rolling velocity of airframe causes the amplitudes of ON-OFF control terms to decrease. This results in limit cycles with smaller width. The proposed analytical formulation may be used in analyzing other dynamic instability resources like conical motion as well as closed loop analysis of one pair ON-OFF actuator.

\section{References}

Cohen, C. J., Clare, T. A., \& Stevens, F. L. (1974). Analysis of the nonlinear rolling motion of finned missiles. AIAA Journal, 12(3), 303-309. https://doi.org/10.2514/3.49225

Dawkins, P. (2011). Differential, equations. Lamar University.

Dormand, J. R., \& Prince, P. J. (1980). A family of embedded Runge-Kutta formulae. Journal of Computational and Applied Mathematics, 6(1), 19-26.

https://doi.org/10.1016/0771-050X(80)90013-3

Eikensberry, R. S. (1970, February). Analysis of the angular motion of missile. In Sandia Laboratory, Technical Report SC-CR-70-6051. Sandia National Laboratories.

Koohmaskan, K., Arvan, M. R., Vali, A. R., \& Farid, B. (2015). Dynamic stability conditions for a rolling flight vehicle applying continuous actuator. Aerospace Science and Technology, 42, 451-458. https://doi.org/10.1016/j.ast.2015.02.007 
Koohmaskan, K., Vali, A. R., Arvan, M. R., \& Farid, B. (2016, March). Analysis of two-position and continuous actuators in rolling airframe control. In 13th Conference of Iranian aerospace society (pp. 4-6). Tehran University, Iran (in Persian).

Lestage, A. (2000). Analysis of control and guidance of rolling missiles with a single plane of control fins. In Proceedings of the AIAA Conference on Guidance, Navigation, and Control (pp. 1-11). Denver, CO. AIAA Inc. https://doi.org/10.2514/6.2000-3971

Li, K., Yang, S., \& Zhao, L. (2012). Stability of spinning missiles with an acceleration autopilot. Journal of Guidance, Control and Dynamics, 35(3), 774-786.

https://doi.org/10.2514/1.56122

Malmgren, A. (1999). Modeling and analysis of rolling missiles with a single control surface plane. In Proceedings of the AIAA Conference on Guidance, Navigation, and Control (pp. 197204). Portland, Oregon. AIAA Inc. https://doi.org/10.2514/6.1999-3977

Mirzaei, M., \& Alishahi, M. M. (2014). Performance investigation of control and guidance system for a spinning flight vehicle with dithering canard. Journal of Modares Mechanical Engineering, 14(7), 169-175 (in Persian).

Mohammadi, B., Arvan, M. R., \& Koohmaskan, Y. (2016, February). Dither in a rolling airframe flight vehicle with a twoposition actuator: An amplitude distribution approach. Transactions of the Institute of Measurement and Control, 39(8), 1205-1215. https://doi.org/10.1177/0142331216631190

Murphy, C. H. (1963, July). Free flight motion of symmetric missiles. Ballistic research laboratories, Report No. 1216. Ballistic Research Laboratory. https://doi.org/10.21236/AD0442757

Murphy, C. H. (1981). Symmetric missile dynamic instabilities. Journal of Guidance, Control and Dynamics, 4(5), 464-471. https://doi.org/10.2514/3.56099

Murphy, C. H. (1971). Response of an asymmetric missile to spin varying through resonance. AIAA Journal, 9(11), 2197-2201. https://doi.org/10.2514/3.50025

\section{Appendix A}

Using proof by induction method, Eq. (27) is proved as follows. According to Eq. (7), for $N=1$, the statement of ideal ON-OFF command is given as:

$$
\begin{aligned}
& \delta_{1}(t)=\delta_{\max }\left(u(t)-u\left(t-\frac{T}{2}\right)\right)-\delta_{\max }\left(u\left(t-\frac{T}{2}\right)-u(t-T)\right)= \\
& \delta_{\max }\left(u(t)-2 u\left(t-\frac{T}{2}\right)+u(t-T)\right) .
\end{aligned}
$$

Using characteristic of Laplace transform we find that

$$
\begin{aligned}
& \mathcal{L}\left[\delta_{1}(t) e^{i p t}\right]=\delta_{\text {max }} \mathcal{L}\left[\left(u(t)-2 u\left(t-\frac{T}{2}\right)+u(t-T)\right) e^{i p t}\right]= \\
& \delta_{\text {max }} \mathcal{L}\left[u(t) e^{i p t}-2 u\left(t-\frac{T}{2}\right) e^{i p\left(t-\frac{T}{2}\right)+i p \frac{T}{2}}+u(t-T) e^{i p(t-T)+i p T}\right]= \\
& \frac{\delta_{\text {max }}}{S-i p}\left(1-2 e^{-\frac{T}{2} S+i p \frac{T}{2}}+e^{-T S+i p T S}\right) .
\end{aligned}
$$

Nicolaides, J. D. (1953, June). On the free flight motion of missiles having slight configurational asymmetries. Ballistic research laboratories, Report No. 858, AD 26405. Ballistic Research Laboratory. https://doi.org/10.21236/AD0026405

Nobahari, H., \& Mohammadkarimi, H. (2012). Multiple-input describing function technique applied to design a single channel ON-OFF controller for a spinning flight vehicle. Journal of Aerospace Engineering, 226(6), 631-645. https://doi.org/10.1177/0954410011414521

Shampine, L. F., \& Reichelt, M. W. (1997). The MATLAB ODE Suite. SIAM Journal on Scientific Computing, 18(1), 1-22. https://doi.org/10.1137/S1064827594276424

Vaughn, H. R. (1968, February). A detailed development of the tricyclic theory. In Sandia laboratory, Technical Report SC-M67-2933. Sandia National Laboratories.

Yan, X., Yang, S., \& Zhang, C. (2010). Coning motion of spinning missiles induced by the rate loop. Journal of Guidance, Control and Dynamics, 33(5), 1490-1499. https://doi.org/10.2514/1.48041

Yan, X., Yang, S., \& Xiong, F. (2011). Stability limits of spinning missiles with attitude autopilot. Journal of Guidance, Control and Dynamics, 34(1), 278-283. https://doi.org/10.2514/1.51627

Zipfel, P. H. (2007). AIAA Education Series (2nd ed.). Modeling and simulation of aerospace vehicles dynamics. AIAA. https://doi.org/10.2514/4.862182

Zhou, W., Yang, S., \& Dong, J. (2013). Coning motion instability of spinning missiles induced by hinge moment. Aerospace Science and Technology, 30(1), 239-245. https://doi.org/10.1016/j.ast.2013.08.008

Zhou, W., Yang, S., \& Zhao, L. (2014, January). Limit circular motion of spinning projectiles induced by backlash of actuators. In AIAA Atmospheric Flight Mechanics Conference (pp. 494-505). National Harbor, Maryland, American Institute of Aeronautics and Astronautics Inc. https://doi.org/10.2514/6.2014-0886

Substituting in Eq. (26) for $N=1$, we get

$$
\begin{aligned}
& \xi_{1}(S)=\frac{K_{1}}{S-\phi_{1}}+\frac{K_{2}}{S-\phi_{2}}+ \\
& \left(\frac{K_{3}}{S-\phi_{1}}+\frac{K_{4}}{S-\phi_{2}}+\frac{K_{5}}{S-i p}\right) \times\left(1-2 e^{i p \frac{T}{2} S}+e^{i p T S}\right),
\end{aligned}
$$

so, Eq. (27) is satisfied for $N=1$. Assuming Eq. (27) is satisfied for $N$, we will try to prove it for $N+1$. For $N+1$, the statement of ideal ON-OFF command is expressed as:

$$
\begin{aligned}
& \delta_{N+1}(t)=\delta_{\max } \sum_{j=0}^{N}\left(u(t-j T)-u\left(t-\frac{2 j+1}{2} T\right)\right)- \\
& \delta_{\max } \sum_{j=1}^{N+1}\left(u\left(t-\frac{2 j-1}{2} T\right)-u(t-j T)\right)= \\
& \delta_{N}(t)+\delta_{\max }\left(u(t-N T)-u\left(t-\frac{2 N+1}{2} T\right)\right)- \\
& \delta_{\max }\left(u\left(t-\frac{2(N+1)-1}{2} T\right)-u(t-(N+1) T)\right) .
\end{aligned}
$$


Substituting in Eq. (26) we find that

$$
\begin{aligned}
& \xi_{N+1}(S)=\xi_{N}(S)+\frac{i M_{c} \delta_{\max }}{S^{2}-\left(H+i I_{p}\right) S+\left(M+i I_{p} T\right)} \times \\
& \left(\begin{array}{l}
\mathcal{L}\left[\left(u(t-N T)-u\left(t-\frac{2 N+1}{2} T\right)\right) e^{i p t}\right]- \\
\left.\mathcal{L}\left[\left(u\left(t-\frac{2(N+1)-1}{2} T\right)-u(t-(N+1) T)\right) e^{i p t}\right]\right),
\end{array}\right.
\end{aligned}
$$

then,

$$
\begin{aligned}
& \xi_{N+1}(S)=\xi_{N}(S)+\frac{i M_{c} \delta_{\max }}{S^{2}-\left(H+i I_{p}\right) S+\left(M+i I_{p} T\right)} \times \\
& \left(\begin{array}{l}
\mathcal{L}\left[u(t-N T) e^{i p(t-N T)+i p N T}-u\left(t-\frac{2 N+1}{2} T\right) e^{i p\left(t-\frac{2 N+1}{2} T\right)+i p \frac{2 N+1}{2} T}\right]- \\
\mathcal{L}\left[u\left(t-\frac{2(N+1)-1}{2} T\right) e^{i p\left(t-\frac{2(N+1)-1}{2} T\right)+i p \frac{2(N+1)-1}{2} T}\right]+ \\
\mathcal{L}\left[u(t-(N+1) T) e^{i p(t-(N+1) T)+i p(N+1) T}\right]
\end{array}\right.
\end{aligned}
$$

Getting Laplace transformation, we have:

$$
\begin{aligned}
& \xi_{N+1}(S)=\xi_{N}(S)+\frac{i M_{c} \delta_{\max }}{\left(S-\phi_{1}\right)\left(S-\phi_{2}\right)} \times \frac{1}{S-i p} \\
& \left(\begin{array}{c}
e^{-N T S+i p N T}-e^{-\frac{2 N+1}{2} T S+i p \frac{2 N+1}{2} T} \\
-e^{-\frac{2 N+1}{2} T S+i p \frac{2 N+1}{2} T}+e^{-(N+1) T S+i p(N+1) T}
\end{array}\right)
\end{aligned}
$$

By writing the explicit expression of $\xi_{N}(S)$ we get:

$$
\begin{aligned}
& \xi_{N+1}(S)=\frac{K_{1}}{S-\phi_{1}}+\frac{K_{2}}{S-\phi_{2}}+ \\
& \left(\frac{K_{3}}{S-\phi_{1}}+\frac{K_{4}}{S-\phi_{2}}+\frac{K_{5}}{S-i p}\right) \times \\
& \left(\begin{array}{c}
1+2 \sum_{j=1}^{N-1} e^{-j T S} e^{i p j T}-2 \sum_{j=0}^{N-1} e^{-\frac{2 j+1}{2} T S} e^{i p \frac{2 j+1}{2} T}+e^{-N T S} e^{i p N T} \\
+e^{-N T S+i p N T}-2 e^{-\frac{2 N+1}{2} T S+i p \frac{2 N+1}{2} T}+e^{-(N+1) T S+i p(N+1) T}
\end{array}\right)
\end{aligned}
$$

At the end we have

$$
\begin{aligned}
& \xi_{N+1}(S)=\frac{K_{1}}{S-\phi_{1}}+\frac{K_{2}}{S-\phi_{2}}+\left(\frac{K_{3}}{S-\phi_{1}}+\frac{K_{4}}{S-\phi_{2}}+\frac{K_{5}}{S-i p}\right) \times \\
& \left(1+2 \sum_{j=1}^{N} e^{-j T S} e^{i p j T}-2 \sum_{j=0}^{N} e^{-\frac{2 j+1}{2} T S} e^{i p \frac{2 j+1}{2} T}+e^{-(N+1) T S+i p(N+1) T}\right)
\end{aligned}
$$

Therefore, Eq. (27) is satisfied for $N+1$, by a result it is satisfied for each $N \in \mathbb{N}$.

\section{Appendix B}

In a similar way, of proving Eq. (27), we will prove Eq. (38) using the mathematical method "Proof in induction" as below steps. First, we prove it for $N=1$. The statement of real ON-OFF command for $N=1$ is given as:

$\delta_{1}(t)=2 \delta_{\max }\left(-e^{-\frac{1}{\tau} t}\left(u(t)-u\left(t-\frac{T}{2}\right)\right)+0.5\right)-$
$2 \delta_{\max }\left(1-e^{-\frac{1}{\tau}\left(t-\frac{T}{2}\right)}\right)\left(u\left(t-\frac{T}{2}\right)-u(t-T)\right)$.

By getting properties of Laplace transformation, we find that

$$
\begin{aligned}
& \mathcal{L}\left[\delta_{1}(t) e^{i p t}\right]=2 \delta_{\max } \mathcal{L}\left[-\left(u(t)-u\left(t-\frac{T}{2}\right)\right) e^{t\left(i p-\frac{1}{\tau}\right)}+0.5 e^{i p t}\right]- \\
& 2 \delta_{\max } \mathcal{L}\left[\left(e^{i p t}-e^{t\left(i p-\frac{1}{\tau}\right)} e^{+\frac{T}{2 \tau}}\right)\left(u\left(t-\frac{T}{2}\right)-u(t-T)\right)\right] .
\end{aligned}
$$

Simplification results in:

$$
\begin{aligned}
& \mathcal{L}\left[\delta_{1}(t) e^{i p t}\right]=2 \delta_{\max }\left(-\frac{1}{S-i p+\frac{1}{\tau}}+\frac{e^{-\frac{T}{2} S} e^{\frac{T}{2}\left(i p-\frac{1}{\tau}\right)}}{S-i p+\frac{1}{\tau}}+\frac{0.5}{S-i p}\right)- \\
& 2 \delta_{\max }\left(\frac{e^{-\frac{T}{2} S} e^{i p \frac{T}{2}}}{S-i p}-\frac{e^{-T S} e^{i p T}}{S-i p}-\frac{e^{-\frac{T}{2} S} e^{\frac{T}{2}\left(i p-\frac{1}{\tau}\right)+\frac{T}{2 \tau}}}{S-i p+\frac{1}{\tau}}+\frac{e^{-T S} e^{T\left(i p-\frac{1}{\tau}\right)+\frac{T}{2 \tau}}}{S-i p+\frac{1}{\tau}}\right) .
\end{aligned}
$$

And finally:

$$
\begin{aligned}
& \mathcal{L}\left[\delta_{1}(t) e^{i p t}\right]=\delta_{\max }\left(\frac{-2}{S-i p+\frac{1}{\tau}}+2 \frac{e^{-\frac{T}{2} S} e^{\frac{T}{2}\left(i p-\frac{1}{\tau}\right)}}{S-i p+\frac{1}{\tau}}+\frac{1}{S-i p}\right)+ \\
& \delta_{\max }\left(-2 \frac{e^{-\frac{T}{2} S} e^{i p \frac{T}{2}}}{S-i p}+2 \frac{e^{-T S} e^{i p T}}{S-i p}+2 \frac{e^{-\frac{T}{2} S+i p \frac{T}{2}}}{S-i p+\frac{1}{\tau}}-2 \frac{e^{-T S} e^{i p T-\frac{T}{2 \tau}}}{S-i p+\frac{1}{\tau}}\right) .
\end{aligned}
$$

Substituting in Eq. (26) for $N=1$, we get

$$
\begin{aligned}
& \xi_{1}(s)=\frac{K_{1}}{S-\phi_{1}}+\frac{K_{2}}{S-\phi_{2}}+\left(\frac{K_{3}}{S-\phi_{1}}+\frac{K_{4}}{S-\phi_{2}}+\frac{K_{5}}{S-i p+\frac{1}{\tau}}\right) \times \\
& \left.-2+2 e^{-\frac{T}{2} S} e^{\frac{T}{2}\left(i p-\frac{1}{\tau}\right)}+2 e^{-\frac{T}{2} S+i p \frac{T}{2}}-2 e^{-T S} e^{i p T-\frac{T}{2 \tau}}\right)+ \\
& \left(\frac{K_{33}}{S-\phi_{1}}+\frac{K_{44}}{S-\phi_{2}}+\frac{K_{55}}{S-i p}\right) \times\left(1-2 e^{-\frac{T}{2} S} e^{i p \frac{T}{2}}+2 e^{-T S} e^{i p T}\right) .
\end{aligned}
$$


So Eq. (38) is satisfied for $N=1$. Then, we assume that Eq. (38) is satisfied for $N$ and we will try to prove it for $N+1$. For $N+1$, the statement of real ON-OFF command is expressed as follows

$$
\begin{aligned}
& \delta_{N+1}(t)=\delta_{\max }\left(-1-2 \sum_{j=0}^{N} e^{-\frac{t-j T}{\tau}}\left(u(t-j T)-u\left(t-\frac{2 j+1}{2} T\right)\right)-\right. \\
& 2 \delta_{\max } \sum_{j=1}^{N+1}\left(1-e^{\frac{-\left(t-\frac{2 j-1}{2} T\right)}{\tau}}\right)\left(u\left(t-\frac{2 j-1}{2} T\right)-u(t-j T)\right) .
\end{aligned}
$$

More simplification gives:

$$
\begin{aligned}
& \delta_{N+1}(\mathrm{t})=\delta_{N}-2 \delta_{\text {max }} e^{-\frac{t-N T}{\tau}}\left(u(t-N T)-u\left(t-\frac{2 N+1}{2} T\right)\right)- \\
& 2 \delta_{\text {max }}\left(1-e^{-\frac{-t-\frac{2 N+1}{2} T}{\tau}}\right)\left(u\left(t-\frac{2 N+1}{2} T\right)-u(t-(N+1) T)\right) .
\end{aligned}
$$

Substituting Eq. (B.7) in Eq. (26) we find that:

$$
\begin{aligned}
& \xi_{N+1}(S)=\xi_{N}(S)+\frac{i M_{c} \delta_{\max }}{\left(S-\phi_{1}\right)\left(S-\phi_{2}\right)} \times \\
& \mathcal{L}\left[-2 e^{-\frac{t-N T}{\tau}}\left(u(t-N T)-u\left(t-\frac{2 N+1}{2} T\right)\right) e^{i p t}\right]- \\
& \left.\mathcal{L}\left[2\left(1-e^{-\frac{t-\frac{2 N+1}{2} T}{\tau}}\right)\left(u\left(t-\frac{2 N+1}{2} T\right)-u(t-(N+1) T)\right) e^{i p t}\right]\right)
\end{aligned}
$$

By taking Laplace transformation of terms in brackets in a similar way to case $N=1$, we get

$$
\left.\begin{array}{l}
\xi_{N+1}(S)=\xi_{N}(S)+\frac{i M_{c} \delta_{\max }}{\left(S-\phi_{1}\right)\left(S-\phi_{2}\right)} \times \\
-2 \frac{e^{i p N T-N T S}}{S-i p+\frac{1}{\tau}}+2 \frac{e^{i p \frac{2 N+1}{2} T-\frac{T}{\tau}-\frac{2 N+1}{2} T S}}{S-i p+\frac{1}{\tau}}-2 \frac{e^{i p \frac{2 N+1}{2} T-\frac{2 N+1}{2} T S}}{S-i p} \\
+2 \frac{e^{i p(N+1) T-(N+1) T S}}{S-i p}+2 \frac{e^{i p \frac{2 N+1}{2} T-\frac{2 N+1}{2} T S}}{S-i p+\frac{1}{\tau}}-2 \frac{e^{i p(N+1) T-(N+1) T S-\frac{T}{2 \tau}}}{S-i p+\frac{1}{\tau}}
\end{array}\right) .
$$

After simplification, we have

$$
\begin{aligned}
& \xi_{N+1}(S)=\xi_{N}(S)+\frac{i M_{c} \delta_{\max }}{\left(S-\phi_{1}\right)\left(S-\phi_{2}\right)\left(S-i p+\frac{1}{\tau}\right)} \times \\
& \left(-2 e^{i p N T-N T S}+2 e^{i p \frac{2 N+1}{2} T-\frac{T}{\tau}-\frac{2 N+1}{2} T S}+2 e^{i p \frac{2 N+1}{2} T-\frac{2 N+1}{2} T S}-2 e^{i p(N+1) T-(N+1) T S-\frac{T}{2 \tau}}\right)+ \\
& \left.\frac{i M_{c} \delta_{\max }}{\left(S-\phi_{1}\right)\left(S-\phi_{2}\right)(S-i p)} \times\left(-2 \frac{e^{i p \frac{2 N+1}{2} T-\frac{2 N+1}{2} T S}}{S-i p}+2 \frac{e^{i p(N+1) T-(N+1) T S}}{S-i p}\right) . \quad \text { (B. } 10\right)
\end{aligned}
$$

Writing the expression of $\xi_{N}(S)$ and adding the rest expressions to the sum term, we get

$$
\begin{aligned}
& \xi_{N+1}(S)=\frac{K_{1}}{S-\phi_{1}}+\frac{K_{2}}{S-\phi_{2}}+\left(\frac{K_{3}}{S-\phi_{1}}+\frac{K_{4}}{S-\phi_{2}}+\frac{K_{5}}{S-i p+\frac{1}{\tau}}\right) \times \\
& \left(-2 \sum_{j=1}^{N+1} e^{-(j-1) T S} e^{i p(j-1) T}+2 \sum_{j=1}^{N+1} e^{-\frac{2 j-1}{2} T S} e^{i p \frac{2 j-1}{2} T}\left(1+e^{\frac{-T}{2 \tau}}\right)-2 \sum_{j=1}^{N+1} e^{-j T S} e^{i p j T-\frac{T}{2 \tau}}\right) \\
& +\left(\frac{K_{33}}{S-\phi_{1}}+\frac{K_{44}}{S-\phi_{2}}+\frac{K_{55}}{S-i p}\right) \times\left(1-2 \sum_{j=1}^{N+1} e^{-\frac{2 j-1}{2} T S} e^{i p \frac{2 j-1}{2} T}+2 \sum_{j=1}^{N+1} e^{-j T S} e^{i p j T}\right) \text {. }
\end{aligned}
$$

Therefore, Eq. (38) is satisfied $\mathrm{f}$ or $N+1$ and it is proved for any $N \in \mathbb{N}$. 